Article

\title{
Bienzymatic Biosensor for Rapid Detection of Aspartame by Flow Injection Analysis
}

\author{
Maria-Cristina Radulescu *, Bogdan Bucur, Madalina-Petruta Bucur and Gabriel Lucian Radu \\ Centre of Bioanalysis, National Institute of Research and Development for Biological Sciences, 296, \\ Splaiul Independentei, Bucharest 060031, Romania; E-Mails: bucurica@yahoo.com (B.B.); \\ madalina_dondoi@yahoo.com (M.-P.B.); rglucian2000@yahoo.com (G.L.R.)
}

* Author to whom correspondence should be addressed; E-Mail: cristinariz2002@yahoo.com; Tel./Fax: +40-021-22-00-900.

Received: 6 November 2013; in revised form: 16 December 2013 / Accepted: 24 December 2013 / Published: 9 January 2014

\begin{abstract}
A rapid, simple and stable biosensor for aspartame detection was developed. Alcohol oxidase (AOX), carboxyl esterase $(\mathrm{CaE})$ and bovine serum albumin (BSA) were immobilised with glutaraldehyde (GA) onto screen-printed electrodes modified with cobalt-phthalocyanine (CoPC). The biosensor response was fast. The sample throughput using a flow injection analysis (FIA) system was $40 \mathrm{~h}^{-1}$ with an RSD of $2.7 \%$. The detection limits for both batch and FIA measurements were $0.1 \mu \mathrm{M}$ for methanol and $0.2 \mu \mathrm{M}$ for aspartame, respectively. The enzymatic biosensor was successfully applied for aspartame determination in different sample matrices/commercial products (liquid and solid samples) without any pre-treatment step prior to measurement.
\end{abstract}

Keywords: alcohol oxidase; carboxyl esterase; flow injection analysis; aspartame

\section{Introduction}

In the last decades, the high-intensity sweeteners have increasingly been used by the food and pharmaceutical industries to improve the taste of different products. Despite the long-term usage of artificial high-intensity sweeteners like aspartame, saccharin, neotame, acesulfame potassium and sucralose, their safety is still debated and currently European Food Safety Agency (EFSA) is conducting a full re-evaluation process of aspartame under the mandate of the European Commission [1]. Toxicological and clinical studies indicate that an excess of artificial sweeteners induces various health 
problems such as memory loss, headaches, seizures, cancer, etc. [2-4]. In consequence, the analysis of sweeteners in foods and pharmaceutical preparations is important for health consumer protection.

Various analytical techniques have been applied in the analysis of natural sugars and artificial sweeteners. High performance liquid chromatography (HPLC) is widely used for the determination of sweeteners [5-7], but this technique is based on expensive equipment, requires long and complex sample pretreatment, uses toxic organic solvents and various reagents. Different alternative analytical methods based on various detections, such as electrochemical [8-10], spectrophotometric [11,12], chemiluminescent [13] or colorimetric detection [14,15] have been developed. Even if these techniques require simple equipment, some of them are time-consuming, involve different chemical reagents, or do not have the necessary selectivity for the analyte determination in relevant commercial samples.

(Bio)sensors are interesting analytical devices with good analytical performance for the rapid analysis of complex samples [16,17]. Only few papers describe biosensors for the determination of aspartame in soft drinks. Those biosensors were based on the chemical co-immobilization of enzymes on different electrodes, such as ammonia-gas-sensing electrode [18], platinum-based hydrogen peroxide electrode [19], oxygen electrode [20], or graphite epoxy composite electrode [21]. Another strategy is based on the enzyme immobilization into columns integrated in flow systems: two enzyme columns containing peptidase and aspartate aminotransferase, respectively, immobilized on activated aminopropyl glass beads and an L-glutamate oxidase electrode [22] or another system consisting of a column containing pronase and an L-amino acid oxidase electrode [23]. These biosensors require long analysis times, show a reduced linear range, short lifetimes, or weak detection limits. Thus, fast, inexpensive methods of analysis with improved selectivity and sensitivity are required to monitor sweeteners in an extensive range of different commercial product matrices.

This paper presents a rapid, simple, inexpensive, and stable bienzymatic amperometric biosensor based on co-immobilization of $\mathrm{AOX}$ and $\mathrm{CaE}$ on cobalt-phtalocyanine modified screen-printed electrodes for the fast and selective aspartame quantification in complex samples. The proposed biosensor was implemented in a FIA system that requires only minimum operator intervention. The FIA system was used for the determination of aspartame in commercial pharmaceutical formulations and food without any pretreatment other than sample solubilisation/dilution with a buffer solution.

\section{Experimental Section}

\subsection{Chemicals and Materials}

Alcohol oxidase AOX (from Hansenula sp. $7.7 \mathrm{UI} / \mathrm{mg}$ solid), carboxyl esterase CaE (from porcine liver, $17 \mathrm{UI} / \mathrm{mg}$ solid), aspartyl phenylalanine methyl ester (aspartame), bovine serum albumin BSA, glutaraldehyde GA (25\% solution in water), potassium phosphate monobasic, sodium phosphate dibasic and potassium chloride were purchased from Sigma-Aldrich (St.Louis, MO, USA). Methanol was obtained from Merck (Darmstadt, Germany). Standard solutions of methanol and aspartame were prepared daily in water.

The amperometric measurements were made with a $0.1 \mathrm{M}$ phosphate buffer solution (PBS) $\mathrm{pH} 7.3$ supplemented with $0.05 \mathrm{M}$ potassium chloride. All aqueous solutions were prepared with purified 
water $\left(18 \mathrm{M} \Omega \cdot \mathrm{cm}^{-1}\right.$; Millipore, Billerica, MA, USA). The soft drinks samples were purchased from a local supermarket. The pharmaceutical formulations were obtained from a local pharmacy.

\subsection{Instruments}

All amperometric measurements were performed using a PGSTAT302N potentiostat/galvanostat (Metrohm-Autolab, Utrecht, The Netherlands) controlled by a PC with the software Nova 1.8. The electromagnetic noise produced by magnetic stirring during batch measurements was reduced using the filter from the ECD module set to $1 \mathrm{~s}$. Cobalt-phthalocyanine (CoPC) screen-printed electrodes were kindly provided by BIOMEM-University of Perpignan, France [24]. An Ag/AgCl pseudoelectrode and a carbon auxiliary electrode were printed alongside with the working electrode. The monocanal FIA manifold was constructed with: a Gilson Minipuls 3 peristaltic pump (Gilson, Middleton, WI, USA), a methacrylate wall-jet flow cell (DropSens, Oviedo, Spain) for electrodes, a sample injection valve (Omnifit, Cambridge, UK) with a $50 \mu \mathrm{L}$ sample loop, connectors and PTFE tubing with $1 \mathrm{~mm}$ i.d.

\subsection{Biosensor Preparation}

The methanol biosensors were prepared as follows: 7.7 IU of AOX was dissolved in $20 \mu \mathrm{L}$ PBS and mixed with $5 \mu \mathrm{L}$ of $0.6 \%$ BSA and $5 \mu \mathrm{L}$ of $1.5 \%$ glutaraldehyde. Four $\mu \mathrm{L}$ of the resulting solution was carefully spread on the surface of the working electrode. The aspartame biosensors were prepared using a similar procedure based on a bienzymatic solution containing 7.7 IU AOX and 18.7 IU CaE in the $20 \mu \mathrm{L}$ PBS. The electrodes were dried at room temperature for $1 \mathrm{~h}$ and then were stored in sealed plastic bags at $-20{ }^{\circ} \mathrm{C}$.

\subsection{Amperometric Measurements}

Aspartame is first cleaved by carboxyl esterase in methanol and a dipeptide: L-Asp-L-Phe. The produced methanol is oxidized by AOX and the resulting hydrogen peroxide is quantified amperometrically (Scheme 1). The batch amperometric measurements were performed in a glass cell containing $5 \mathrm{~mL}$ of PBS under constant magnetic stirring. The current intensity was measured under a constant potential of $+600 \mathrm{mV}$ vs. the screen-printed $\mathrm{Ag} / \mathrm{AgCl}$ pseudoreference electrode. Approximately $60 \mathrm{~s}$ are necessary for the baseline stabilization. After the sample injection in the cell the signal was recorded and a plateau was reached in $20 \mathrm{~s}$, but the total analysis time includes also the cellule replacement and biosensor washing with distilled water. The analytical signal is the difference between the current intensities of the plateau and baseline. To reduce the errors and the analysis time the developed biosensor was used also in a FIA system. The sample was injected in the PBS carrier flow $0.2 \mathrm{~mL} / \mathrm{min}$ using the valve and transported to the flow cell. 
Scheme 1. The aspartame detection mechanism. Note that a part of the enzymatically produced methanol and hydrogen peroxide diffuses towards solution and is not oxidized at the electrode surface.

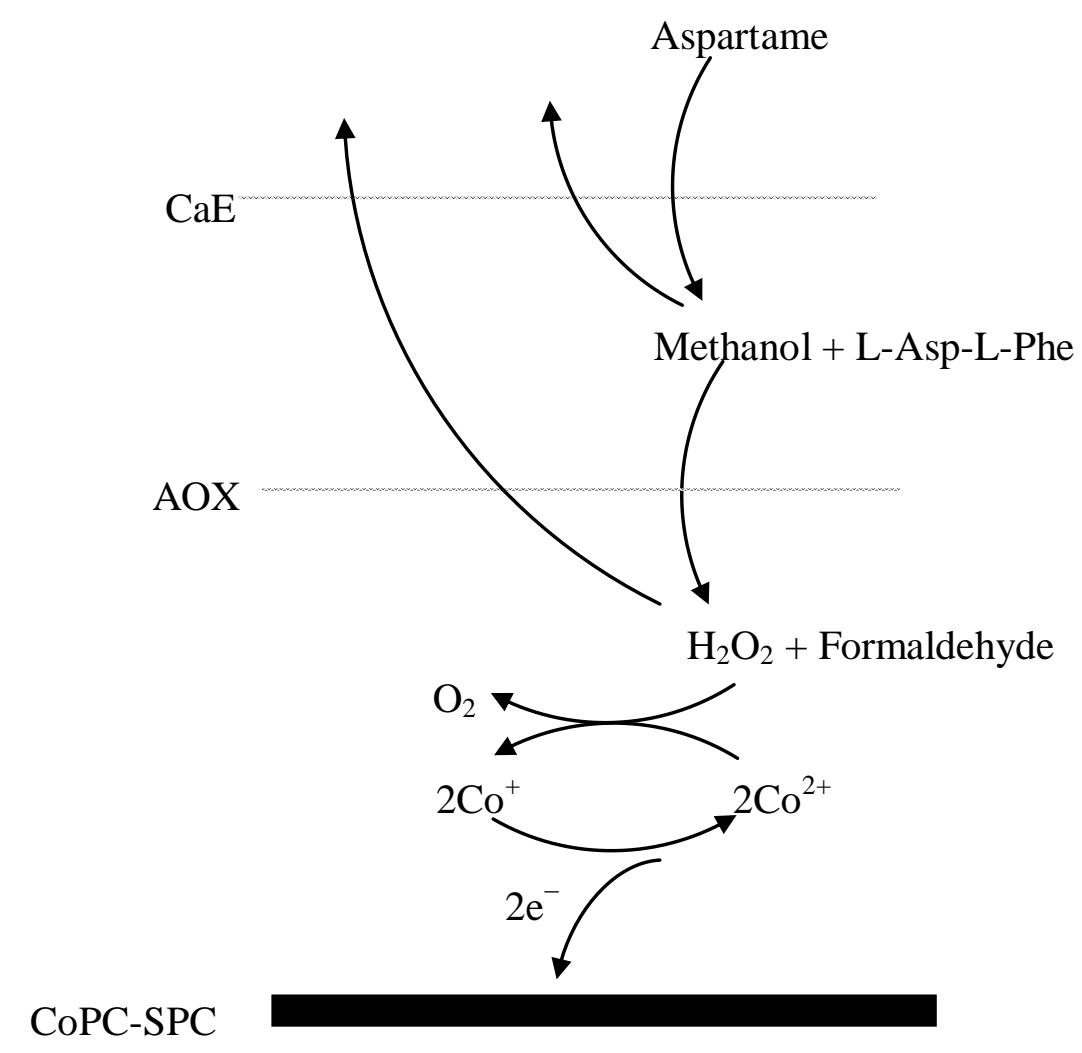

\section{Results and Discussion}

\subsection{Biosensor Optimization}

The initial optimization was carried out in batch mode. The biosensor was tested for both methanol and aspartame detection in order to optimize the working conditions and investigate the kinetics of the mono and bi-enzymatic processes. The optimum volume of enzymes/GA solution that covers the entire working electrode area is $4 \mu \mathrm{L}$. The optimum final concentrations of GA $(0.25 \%)$ and BSA $(0.1 \%)$ were chosen to obtain a stable immobilization of the enzymes. A decrease of the analytical signal was observed for higher concentrations of BSA and/or GA. The cobalt-phthalocyanine mediator can be used for hydrogen peroxide detection at different potentials ranging from $+450 \mathrm{mV}$ [25] to $+600 \mathrm{mV}$ [26]. The higher potentials allow better measurements sensibility, but are prone to interferences. We have chosen the working potential of $600 \mathrm{mV}$ in order to achive a high measurement sensitivity, but for specific samples the potential can be reduced. A study of the $\mathrm{pH}$ influence on the analytical signal obtained using the bi-enzymatic electrode was studied in the range of 6-8 and the optimum value was 7.3. The optimum working conditions for both $\mathrm{CaE}$ and $\mathrm{AOX}$ are similar and the enzymes may be used together. The rapid responses of the developed biosensor were registered in less than $20 \mathrm{~s}$ under the optimum working conditions for both methanol and aspartame as it shown in Figure 1. 
Figure 1. Analytical signals of the bienzymatic electrode recorded in batch for a concentration of $20 \mu \mathrm{M}$ methanol (a) and $20 \mu \mathrm{M}$ aspartame (b), respectively, under the optimum working conditions.

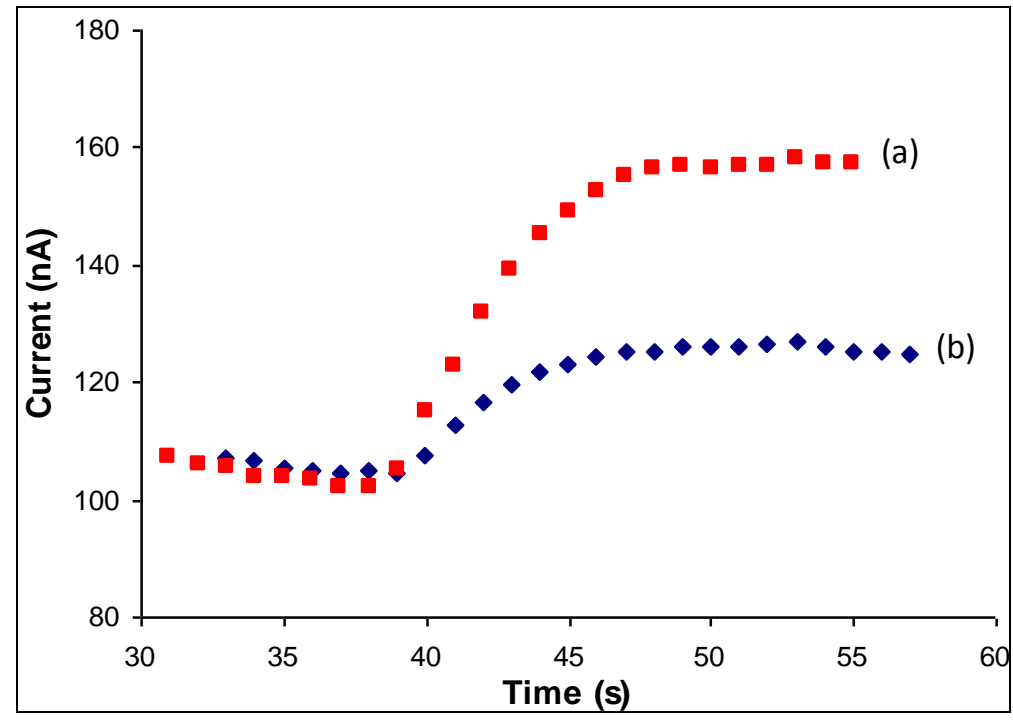

The AOX activity was first optimized for methanol detection and a 1 IU of AOX was chosen in order to obtain a satisfactory measurement sensitivity $(1.887 \mathrm{nA} / \mu \mathrm{M}$ methanol) and detection limit $(0.1 \mu \mathrm{M}$ methanol). The AOX activity is significantly lower in comparison with 20 IU AOX used in other papers [20,21]. The optimum CaE activity per electrode was chosen as $2.5 \mathrm{IU}$ based on the satisfactory sensitivity $(0.825 \mathrm{nA} / \mu \mathrm{M}$ aspartame $)$ and detection limit (0.2 $\mu \mathrm{M}$ aspartame). The lower sensitivity obtained for aspartame detection in comparison with methanol detection is explained by the fact that not all the enzymatically produced methanol is further oxidized by AOX because a part of the methanol is diffusing toward electrochemical cell (Scheme 1). Nevertheless, the magnitude of the analytical signals is sufficient for the aspartame detection. Bare and monoenzymatic electrodes (modified only with $\mathrm{CaE}$ or $\mathrm{AOX}$ ) do not respond to aspartame.

\subsection{Analytical Figures of Merit}

The biosensor response was studied in the concentrations range of $0-1,000 \mu \mathrm{M}$ for methanol and aspartame, respectively. Under the optimum working conditions, a large linear domain was obtained for both aspartame and methanol (Figure 2). The calibration plot for aspartame determination in bach was characterized by a linear domain of concentrations between 5 and $600 \mu \mathrm{M}$ with the equation $\mathrm{I}(\mathrm{nA})=0.8254 x$ Conc aspartame $(\mu \mathrm{M})+9.1294$ and $R^{2}=0.9989$. The calibration plot for methanol determination was linear between 2 and $200 \mu \mathrm{M}$ and was defined by the regression equation. $\mathrm{I}(\mathrm{nA})=1.8872 x$ Conc methanol $(\mu \mathrm{M})+1.5346$ and $\mathrm{R}^{2}=0.9965$. The obtained detection limits (calculated as three times standard deviation of the blank) were $0.2 \mu \mathrm{M}$ for aspartame and $0.1 \mu \mathrm{M}$ for methanol, respectively. 
Figure 2. The calibration plots obtained for methanol (a) and aspartame (b) by using the bienzymatic biosensor in batch.

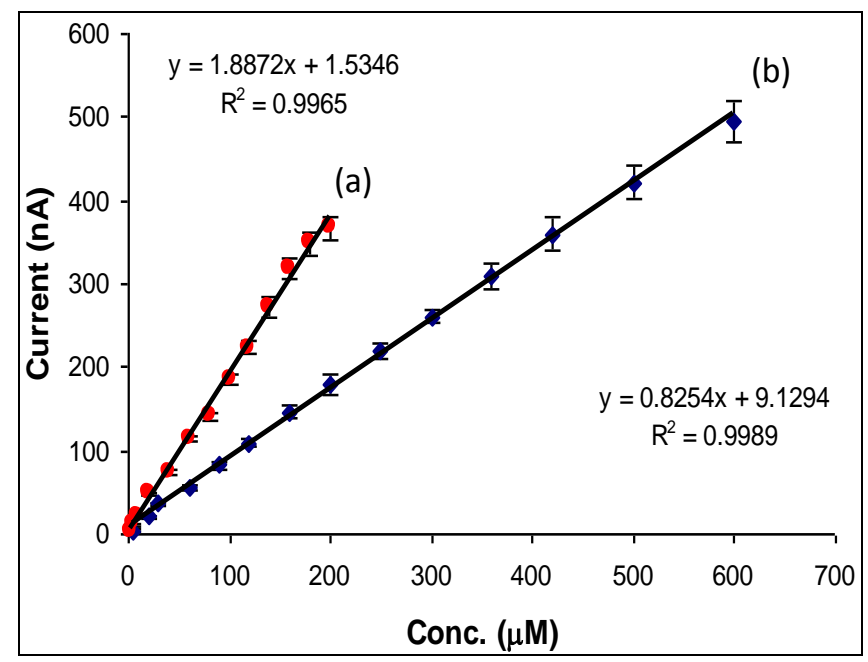

The operational stability of a biosensor was measured by successive measurements of $20 \mu \mathrm{M}$ aspartame and $20 \mu \mathrm{M}$ methanol. The relative standard deviation (RSD) calculated for 10 successive batch measurements was $3.1 \%$ for methanol (53.4 $\mathrm{nA} \pm 1.66)$ and $5.5 \%$ for aspartame $(22.3 \mathrm{nA} \pm 1.45)$. More than 20 successive measurements can be performed with the same electrode without any significant decrease of the analytical signal. The reproducibility of the analytical signals recorded for the injection of $100 \mu \mathrm{M}$ aspartame using different biosensors produced in the same lot was $6 \%$ $(76.7 \pm 4.58 \mathrm{nA}, n=6)$. The biosensors were stored in sealed plastic bags at $-20{ }^{\circ} \mathrm{C}$. A decrease of the signal up to $40 \%$ was observed after 1 month.

The calibration graphs for aspartame were similar in FIA and batch systems. The linear domain of concentrations was between 5 and $600 \mu \mathrm{M}$ with the equation $\mathrm{I}(\mathrm{nA})=0.6886 \mathrm{x}$ Conc aspartame $(\mu \mathrm{M})+$ 12.478 and $\mathrm{R}^{2}=0.9924$ (Figure 3). The relative standard deviation for a concentration of $100 \mu \mathrm{M}$ aspartame by using the FIA system was $2.7 \%$, smaller than in batch. In the same time, the number of measurements was increased to 40 measurements/hour.

Figure 3. The calibration graph obtained for aspartame in FIA (A) and signals (B).
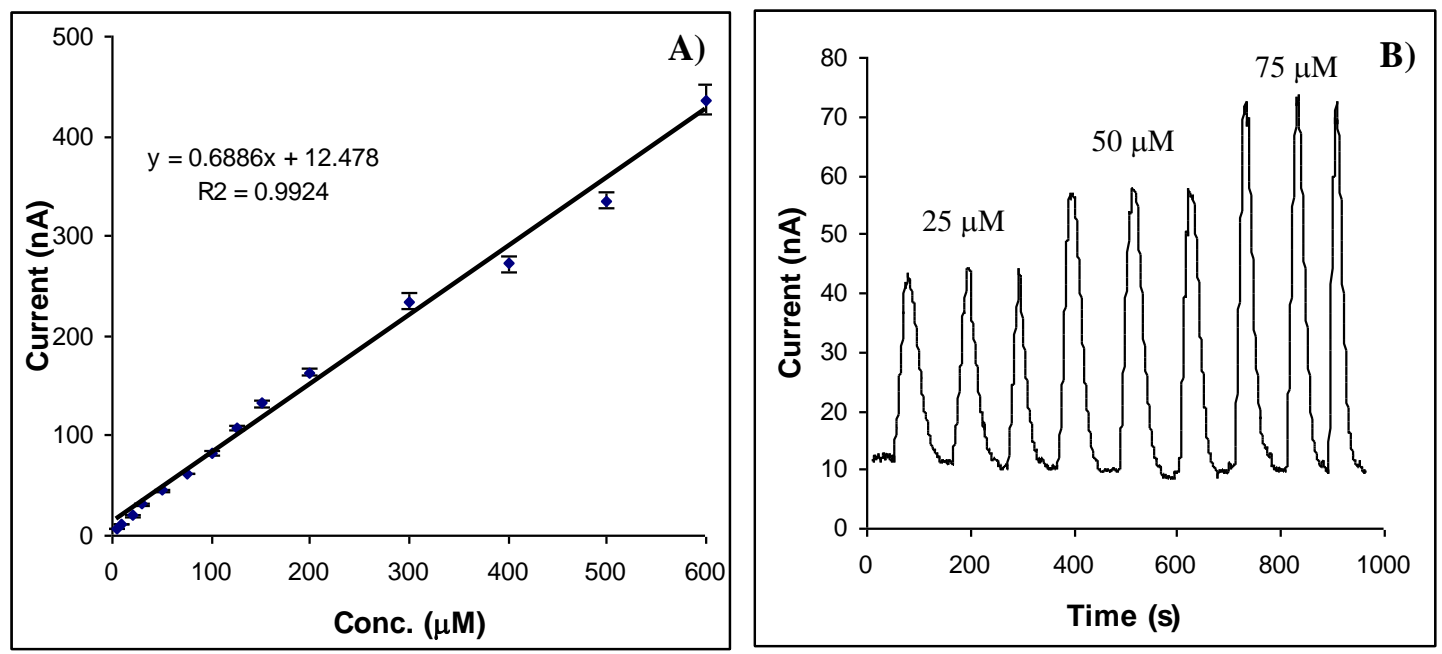
The performances of our biosensor are better or comparable with previous developed biosensors (Table 1). Our biosensor is simple, presents the shortest response time and has a good LOD and RSD. The obtained LOD is sufficient for the analysis of the expected aspartame concentrations in real food samples. The developed biosensor coupled with a simple FIA system has the advantages of a significant reduction of the analysis time in comparison with other systems (e.g., the biosensor developed by Odaci et al. requires $10 \mathrm{~min}$ [20], and of the reduction of the errors and the samples/reagents volume.

Table 1. Comparison of various enzymatic biosensors for aspartame.

\begin{tabular}{|c|c|c|c|c|c|}
\hline Enzymes & $\begin{array}{c}\text { Linear Range } \\
(\mu \mathrm{M})\end{array}$ & $\begin{array}{l}\text { LOD } \\
(\mu \mathrm{M})\end{array}$ & $\begin{array}{c}\text { RSD } \\
(\%)\end{array}$ & $\begin{array}{c}\text { Analysis Time } \\
(\min )\end{array}$ & References \\
\hline Carboxyl esterase and alcohol oxidase & $0.05-0.4$ & - & 2.4 & 10 & {$[20]$} \\
\hline Carboxyl esterase and alcohol oxidase & $2.5-400$ & - & 3.9 & 3 & [21] \\
\hline $\begin{array}{l}\text { Peptidase, aspartate aminotransferase and } \\
\text { glutamate oxidase }\end{array}$ & Up to 1,000 & 20 & 2.2 & $7-8$ & [22] \\
\hline Chymotrypsin and alcohol oxidase & $85-1,200$ & 7.3 & 3.0 & 4 & [27] \\
\hline $\begin{array}{l}\text { Aspartame hydrolyzing enzyme, aspartate } \\
\text { aminotransferase and glutamate oxidase }\end{array}$ & $200-1,500$ & 150 & - & $2-3$ & [28] \\
\hline $\begin{array}{l}\text { Peptidase, aspartate aminotransferase and } \\
\text { glutamate oxidase }\end{array}$ & Up to 200 & 25 & 4.6 & $10-30$ & [29] \\
\hline Carboxyl esterase and alcohol oxidase & $5-600$ & 0.2 & 5.5 & $\begin{array}{l}\text { Less than } 2 \\
\text { (for FIA) }\end{array}$ & This work \\
\hline
\end{tabular}

\subsection{Interferences Study}

Different potentially interfering substances e.g., citric acid, phosphoric acid, glucose, fructose, sucrose, caffeine, L-phenylalanine and sodium benzoate were investigated based on their possible presence in food and pharmaceutical samples. The initial study was carried out using an electrode without enzymes to measure the electrochemical interferences. Subsequently, the interferences study was carried out using bienzymatic biosensors for a methanol concentration of $20 \mu \mathrm{M}$, respectively aspartame and interference of $40 \mu \mathrm{M}$. When the interfering substance was added in the cell no analytical signal, was recorded. After the baseline stabilization, the methanol/aspartame concentration was added in the cell and the corresponding current value was registered. The biosensor response for methanol/aspartame was measured three times in the presence of each interfering substance and the mean value of the response variation was represented. The biosensor response varied between $94 \%$ and $112 \%$ in the presence of the studied interferences (Figure 4). These results must be compared to the measurement errors (5.5\% mentioned in Section 3.2.) and demonstrate that the interferences have only minor effect on the biosensor's response. 
Figure 4. The relative response variation of the bienzymatic biosensor for a concentration of $20 \mu \mathrm{M}$ methanol and $20 \mu \mathrm{M}$ aspartame, respectively, in the absence and the presence of interferences.

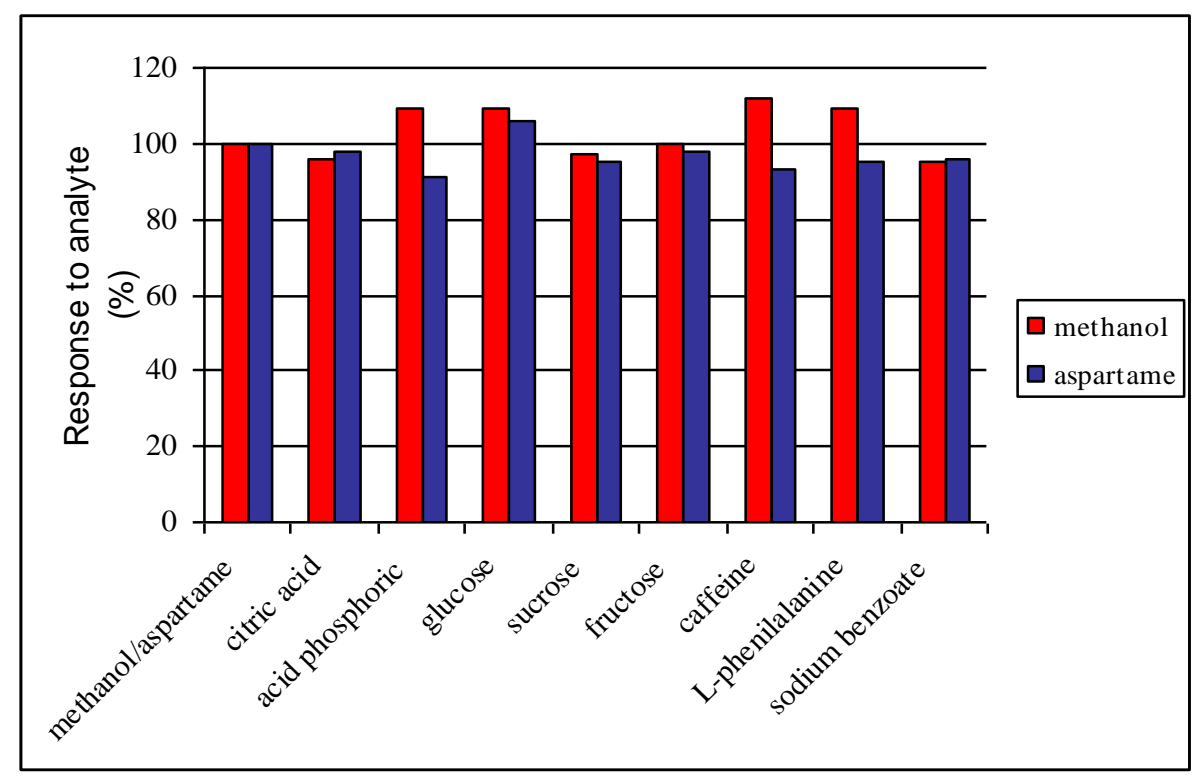

\subsection{Real Sample Analysis}

The developed screen-printed biosensors were tested for the aspartame determination in different sample matrixes: soft drinks and commercial pharmaceutical formulations.

The soft drink brands, Coca-Cola and Pepsi Cola, were manufactured and marketed in Romania and can be classified in two categories: diet drinks and light drinks. These types of drinks mostly consist of carbonated water, sweeteners (aspartame and acesulfame-K), colorants, citric acid, sodium benzoate, phosphoric acid, food additives such as preservatives and flavors. The exact content of aspartame in such drinks was not mentioned on the label. Two pharmaceutical formulations of the same drug (Erdomed single dose sachet and bottle with granules for suspension) were analyzed. The samples contain the same active substance (erdosteine) and several excipients: aspartame, sugar, sodium benzoate, sodium amidonglycolate and flavors. The concentration of aspartame was labelled. The solid pharmaceutical samples were first dissolved in $100 \mathrm{~mL}$ water. Then all the samples (drinks and pharmaceuticals) were analyzed after adequate dilution in PBS without preliminary preparation such as concentration or extraction. Three aliquots of each sample were analyzed and interpolated in the calibration graph. The results for the aspartame amount founded in real samples are given in the Table 2. The obtained concentration levels of aspartame in the analyzed drink samples were found to vary between 58 and $152 \mathrm{mg} / \mathrm{L}$ and they did not exceed the maximum legally allowed UE concentration of $600 \mathrm{mg} / \mathrm{L}$ [1]. In addition, the concentrations of aspartame founded in commercial pharmaceutical formulations were in good agreement with those declared by the producer. The samples were spiked with $50 \mu \mathrm{M}$ aspartame and the obtained recoveries were in the range of $94.1 \%-106.2 \%$. 
Table 2. Analysis of aspartame in real samples.

\begin{tabular}{cccc}
\hline Sample & $\begin{array}{c}\text { Aspartame Concentration } \\
(\mathbf{m g} / \mathbf{L}) \pm \mathbf{S D}^{\mathbf{a}}\end{array}$ & $\begin{array}{c}\text { Label Claimed } \\
\text { Concentration }(\mathbf{m g} / \mathbf{L})\end{array}$ & $\begin{array}{c}\text { Recovery of Spiked } \\
\text { Aspartame (\%) }\end{array}$ \\
\hline Pepsi max classic & 0 & - & 96.82 \\
Pepsi max no sugar & $129 \pm 5.2$ & $\mathrm{n} .1^{\mathrm{b}}$ & 103.77 \\
Pepsi light & $152 \pm 7.0$ & $\mathrm{n} .1$. & 104.13 \\
Coca-cola classic & 0 & $\mathrm{n} .1$. & 94.86 \\
Coca-cola light & $82 \pm 3.1$ & $\mathrm{n} .1$. & 103.54 \\
Coca-cola zero & $58 \pm 2.2$ & $\mathrm{n} .1$. & 106.21 \\
Erdomed 175 & $7,512 \pm 288$ & 8,000 & 94.63 \\
Erdomed 225 & $466 \pm 19$ & 500 & 96.13 \\
\hline
\end{tabular}

Note: ${ }^{a}$ All results are expressed as average \pm standard deviation of three determinations; ${ }^{b}$ n.l., not labeled.

\section{Conclusions}

A rapid and reliable bienzymatic biosensor based on the enzymes $\mathrm{AOX}$ and $\mathrm{CaE}$ was developed for aspartame determination. The detection limits were $0.1 \mu \mathrm{M}$ for methanol and $0.2 \mu \mathrm{M}$ for aspartame, respectively. An improvement of the analysis time and measurement errors was achieved by coupling the biosensor with a simple FIA system. The response of the biosensor was very fast, $20 \mathrm{~s}$ are necessary to obtain the maximum response for methanol and aspartame concentrations, respectively. The developed system was successfully applied to the aspartame determination content in complex samples: soft drinks and commercial pharmaceutical formulations without the pre-treatment of the samples prior the analysis.

\section{Acknowledgments}

This work was supported by the grants of the Romanian National Authority for Scientific Research, CNCS-UEFISCDI (projects numbers: PN-II-RU-TE-2010-100 and PN-II-RU-TE-2011-3-0076). The authors thank Jean-Louis Marty (IMAGES-University of Perpignan Via Domitia, France) for the screen printed electrodes.

\section{Conflicts of Interest}

The authors declare no conflict of interest.

\section{References}

1. Aspartame. Available online: http://www.efsa.europa.eu/en/topics/topic/aspartame.htm?wtrl=01 (accessed on 23 August 2013).

2. Magnuson, B.A.; Burdock, G.A.; Doull, J.; Kroes, R.M. Aspartame: A safety evaluation based on current use levels, regulations, and toxicological and epidemiological studies. Crit. Rev. Toxicol. 2007, 37, 629-726.

3. Mattes, R.D.; Popkin, B.M. Nonnutritive sweetener consumption in humans: Effects on appetite and food intake and their putative mechanisms. Am. J. Clin. Nutr. 2009, 89, 11-14. 
4. Tandel, K.R. Sugar substitutes: Health controversary over perceived benefits. J. Pharmacol. Pharmacother. 2011, 2, 236-243.

5. Chen, Q.C.; Wang, J. Simultaneous determination of artificial sweeteners, preservatives, caffeine, theobromine and theophylline in food and pharmaceutical preparations by ion chromatography. J. Chromatogr. A 2001, 937, 57-64.

6. Zhu, Y.; Guo, Y.Y.; Ye, M.L.; James, F.S. Separation and simultaneous determination of four artificial sweeteners in food and beverages by ion chromatography. J. Chromatogr. A 2005, 1085, 143-146.

7. Zygler, A.; Wasik, A.; Kot-Wasik, A.; Namiesnik, J. Determination of nine high-intensity sweeteners in various foods by high-performance liquid chromatography with mass spectrometric detection. Anal. Bioanal. Chem. 2011, 400, 2159-2172.

8. Carloni, J.; Santini, A.O.; Nasser, A.L.M.; Pezza, H.R.; de Oliveira, J.E.; Melios, C.B.; Pezza, L. Potentiometric determination of saccharin in commercial artificial sweeteners using a silver electrode. Food. Chem. 2003, 83, 297-301.

9. Pierini, G.D.; Llamas, N.E.; Fragoso, W.D.; Lemos, S.G.; di Nezio, M.S.; Centurion, M.E. Simultaneous determination of acesulfame-K and aspartame using linear sweep voltammetry and multivariate calibration. Microchem. J. 2013, 106, 347-350.

10. Nikolelis, D.P.; Pantoulias, S.; Krull, U.J.; Zeng, J. Electrochemical transduction of the interactions of the sweeteners acesulfame-K, saccharin and cyclamate with bilayer lipid membranes (BLMs). Electrochim. Acta. 2001, 46, 1025-1031.

11. Capitan-Vallvey, F.; Valencia, M.C.; Nicolas, E.A. Flow-through spectrophotometric sensor for the determination of aspartame in low-calorie and dietary products. Anal. Sci. 2004, 20, 1437-1442.

12. Rocha, F.; Rodenas-Torralba, E.; Morales-Rubio, A.; de la Guardia, M. A clean method for flow injection spectrophotometric determination of cyclamate in table sweeteners. Anal. Chim. Acta 2005, 547, 204-208.

13. Niu, W.; Kong, H.; Wang, H.; Zhang, Y.; Zhang, S.; Zhang, X.A. Chemiluminescence sensor array for discriminating natural sugars and artificial sweeteners. Anal. Bioanal. Chem. 2012, 402, 389-395.

14. Musto, C.J.; Lim, S.H.; Suslick, K.S. Colorimetric detection and identification of natural and artificial sweeteners. Anal. Chem. 2009, 81, 6526-6533.

15. Lim, S.H.; Musto, C.J.; Park, E.; Zhong, W.; Suslick, K.S. A colorimetric sensor array for detection and identification of sugars. Org. Lett. 2008, 10, 4405-4408.

16. Mei, Q.S.; Zhang, Z.P. Photoluminescent graphene oxide ink to print sensors onto microporous membranes for versatile visualization bioaasays. Angew. Chem. Int. Ed. 2012, 51, 5602-5606.

17. Zhang, K.; Zhou, H.B.; Mei, Q.S.; Wang, S.H.; Guan, G.J.; Liu, R.Y.; Zhang, J.; Zhang, Z.P. Instant visual detection of trinitrotoluene particulates on various surfaces by ratiometric florescence of dual-emission quantum dots hybrid. J. Am. Chem. Soc. 2011, 133, 8424-8427.

18. Fatibello-Fihlo, O.; Suleiman, A.A.; Guibault, G.G.; Lubrano, G.J. Bienzymatic electrode for the determination of aspartame in dietary products. Anal. Chem. 1988, 60, 2397-2399.

19. Compagnone, D.; O'sullivan, D.; Guilbault, G.G. Amperometric bienzymic sensor for aspartame. Analyst 1997, 122, 487-490. 
20. Odaci, D.; Timur, S.; Telefoncu, A. Carboxyl esterase-alcohol oxidase based biosensor for the aspartame determination. Food Chem. 2004, 84, 493-496.

21. Kirgöz, U.A.; Odaci, D.; Timur, S.; Merkoçi, A.; Alegret, S.; Beşün, N.; Telefoncu, A. A biosensor based on graphite epoxy composite electrode for aspartame and ethanol detection. Anal. Chim. Acta 2006, 570, 165-169.

22. Male, K.B.; Luong, J.H.T.; Mulchandani, A. Determination of aspartame in dietary food products by a FIA biosensor. Biosens. Bioelectron. 1991, 6, 117-123.

23. Male, K.B.; Luong, J.H.T.; Gibbs, B.; Konishi, Y. An improved FIA biosensor for the determination of aspartame in dietary food products. Appl. Biochem. Biotech. 1993, 38, 189-201.

24. Li, Z.H.; Guedri, H.; Vigier, B.; Sun, S.G.; Marty, J.L. Optimization of hydrogen peroxide detection for methyl mercaptan biosensor. Sensors 2013, 13, 5028-5039.

25. Kenneth, I.O.; Zhixin, Z.; Tebello, N.; Immobilized cobalt(II) phthalocyanine-Cobalt(II) porphyrin pentamer at a glassy carbon electrode: Applications to efficient amperometric sensing of hydrogen peroxide in neutral and basic media. Electrochem. Commun. 2005, 7, 679-684.

26. Philani, N.M.; Kenneth, I.O.; Tebello, N. Tetracarboxylic acid cobalt phthalocyanine SAM on gold: Potential applications as amperometric sensor for $\mathrm{H}_{2} \mathrm{O}_{2}$ and fabrication of glucose biosensor. Electrochim. Acta 2006, 52, 177-186.

27. Pena, R.M.; Lima, L.F.; Saraiva, M.L. Sequential injection analysis-based flow system for the enzymatic determination of aspartame. Anal. Chim. Acta 2004, 514, 37-43.

28. Villarta, R.L.; Suleiman, A.A.; Guilbault, G.G. Amperometric enzyme electrode for the determination of aspartame in diet food. Microchem. J. 1993, 48, 60-64.

29. Mulchandani, A.; Male, K.B.; Luong, J.H.T.; Gibbs, B.F. Enzymatic assay technique for the determination of aspartame. Anal. Chim. Acta 1990, 234, 465-469.

(C) 2014 by the authors; licensee MDPI, Basel, Switzerland. This article is an open access article distributed under the terms and conditions of the Creative Commons Attribution license (http://creativecommons.org/licenses/by/3.0/). 